\title{
détermination d'une loi de comportement pour le cisaillement des sols pulvérulents
}

\author{
application au calcul d'essais triaxiaux*
}

\author{
par \\ J. Monnet \\ Assistant à l'Institut Universitaire de Technologie $n^{\circ} 1$ de Lyon \\ J. Gielly \\ Maître de Conférence à l'Institut Universitaire de Technologie $n^{\circ} 1$ de Lyon
}

Les ouvrages de Génie Civil en contact avec un sol sont, du fait de leur complexité croissante, de plus en plus difficiles à calculer. Les caractéristiques mécaniques du sol de fondation, sa géométrie, les différentes sollicitations auxquelles il est soumis, sont autant de variables difficiles à prendre en compte dans les calculs classiques. Une simplification de la réalité consiste à diviser le système réel en un ensemble de petits éléments de dimensions finies, dont les caractéristiques sont connues. L'utilisation de l'ordinateur permeț de juxtaposer l'action de tous les éléments et de calculer les contraintes et les déformations en tout point du système.

En mécanique des sols, les calculs sont classiquement menés en admettant que le massif concerné est soit totalement en équilibre élastique, soit totalement en équilibre limite plastique.

Le calcul par éléments finis devrait permettre d'aborder l'étude du comportement réel du massif, en prenant en compte, en tout point, la loi effort-déformation du sol, notamment lors du passage de l'état élastique à l'état plastique.

C'est à partir de la théorie proposée par Duncan et Chang (1970) que la méthode des éléments finis s'est étendue au calcul d'ouvrages : stabilité de pentes, barrages en terre ou écluses. Les études plus récentes de Darve (1974) et Chambon (1975) ont ensuite permis de prendre en compte un comportement plus réel du sol, notamment au niveau de la dilatance. Le calcul se déroule de façon incrémentale. Les incréments de contrainte et de déformation qui surviennent au cours d'un chargement, sont calculés à l'aide d'une matrice de rigidité générale. Cette matrice est fonction de l'incrément et doit être recalculée à chaque étape, ce qui demande beaucoup de temps de calcul.

Par ailleurs, le comportement plastique du sol a fait l'objet de l'article de Rowe (1962) qui a abouti à des formules exprimant le comportement du sol. L'auteur s'intéresse au rapport des contraintes principales majeures et mineures. A l'aide d'une modélisation du contact entre sphères, il exprime l'énergie de déformation du milieu granulaire,

- Cette recherche a été effectuée au laboratoire de mécanique des sols de l'Institut National de Sciences Appliquées (INSA) de Lyon. mais en conservant toujours, comme paramètre principal, le rapport des contraintes. C'est donc une approche particulière qui donne des résultats corrects dans le cas de l'essai triaxial de révolution.

Une autre approche du phénomène de plasticité a été effectuée par l'Ecole de Cambridge d'abord sur l'argile. puis sur le sable par Poorooshasb, Holubec et Sherbourne (1966 et 1967). Les auteurs expriment l'énergie de déformation du sol de façon globale, mais sans faire intervenir le frottement intergranulaire. Le calcul théorique d'un essai triaxial nécessite la réalisation d'une série d'essais triaxiaux, si bien que l'utilisation pratique de la loi de comportement est difficile.

L'étude de Frydman, Zeitlen, Alpan (1973) et Frydman (1974) est plus intéressante encore puisque les auteurs expriment l'énergie de déformation du sol en faisant intervenir le frottement intergranulaire. Ils aboutissent alors à une formule valable dans tous les cas de chargement, et qui utilise un seul paramètre.

Cette dernière formulation théorique est particulièrement adaptable aux besoins de calcul de l'ingénieur. Nous avons décidé de l'analyser dans l'optique d'une adaptation à un programme de calcul par éléments finis. Le but était de créer un modèle capable de représenter le comportement du sol de façon précise, mais également exigeant un minimum de données particulières à la théorie choisie, de façon à ce que l'ingénieur soit capable d'utiliser le programme informatique.

La théorie que nous développons est globale. Elle n'est plus incrémentale, et demande par conséquent moins de temps de calcul sur ordinateur, et donc moins de dépense qu'une autre. Nous calculons enfin une série d'essais triaxiaux de révolution dans un but de vérification.

\section{La loi de comportement de Frydman et al.}

Ces auteurs ont montré, à partir de l'analyse de nombreux essais triaxiaux réalisés sur des sols pulvérulents, qu'il apparaît trois phases différentes du comportement du sol au cours de la déformation en distorsion, c'est-à-dire avec une contrainte moyenne constante ou variant peu.

a) Dans le domaine des faibles sollicitations, le sol est assimilable à un solide élastique avec une précision suffisante. Les variations de volume sont faibles. 
b) A partir d'une valeur constante du frottement dans le plan octaédrique, il apparaît des déformations plastiques d'écrouissage; des gonflements sont alors observés.

Frydman et al. ont montré que ce seuil de déformation plastique est donné par:

$$
-\frac{\tau_{\text {oct }}}{\sigma_{\text {oct }}}=\operatorname{tg} \varphi_{\mu}
$$

avec

$\tau_{\text {oct }}=\frac{1}{3} \sqrt{\left(\sigma_{1}-\sigma_{2}\right)^{2}+\left(\sigma_{2}-\sigma_{3}\right)^{2}+\left(\sigma_{3}-\sigma_{1}\right)^{2}}$

et $\sigma_{\text {oct }}=\frac{1}{3}\left(\sigma_{1}+\sigma_{2}+\sigma_{3}\right)$

$\sigma_{1}, \sigma_{2}, \sigma_{3}$ sont les contraintes principales prises négatives en compression. $\tau_{\text {oct }}$ et $\sigma_{\text {oct }}$ sont les composantes de la contrainte dans le
plan octaédrique.

$\sigma_{\text {oct }}$ est aussi appelée : contrainte moyenne.

$\varphi_{\mu} \quad$ est l'angle de frottement grain sur grain du matériau.

Pour l'essai triaxial, nous avons:

$\sigma_{\text {oct }}=\frac{\sigma_{\mathrm{a}}+2 \sigma_{\mathrm{r}}}{3}$ et $\tau_{\text {oct }}=\frac{\left(\sigma_{\mathrm{r}}-\sigma_{\mathrm{a}}\right)}{3} \sqrt{2}$

avec :

$\sigma_{a}$ : contrainte axiale

$\sigma_{\mathrm{r}}:$ contrainte radiale

$\sigma$ varie au cours de l'essai triaxial et la théorie de Frydman est exacte pour $\sigma$ constant, mais nous allons voir que ceci ne perturbe pas de façon significative les calculs informatiques.

Frydman et al. supposent ensuite que l'énergie de déformation plastique est due uniquement à un frottement intergranulaire de valeur $\varphi_{\mu}$, dans le plan octaédrique. On démontre que :

$\frac{\tau_{\text {oct }}}{\sigma_{\text {oct }}}=-\operatorname{tg} \varphi_{\mu}-\frac{d \varepsilon_{\text {oct }}^{p}}{d \varepsilon_{\text {oct }}^{D}}$ (2)

avec $d \varepsilon_{\text {oct }}^{\prime}=\frac{1}{3} \sqrt{\left(d \varepsilon_{1}^{p}-d \varepsilon_{2}^{p}\right)^{2}+\left(d \varepsilon_{2}^{p}-d \varepsilon_{3}^{p}\right)^{2}+\left(d \varepsilon_{3}^{p}-d \varepsilon_{1}^{p}\right)^{2}}$

$d \varepsilon_{\text {oct }}^{p}=\frac{1}{3}\left(d \varepsilon_{1}^{p}+d \varepsilon_{2}^{p}+d \varepsilon_{3}\right)$

$\mathrm{d} \varepsilon_{1}^{p}, \mathrm{~d} \varepsilon_{2}^{p}, \mathrm{~d} \varepsilon_{3}^{p}$ sont les incréments de déformation plastique principale.

Au cours de l'essai triaxial, on a :

$d \varepsilon_{\text {oct }}^{p}=\frac{d \varepsilon_{a}^{p}+2 d \varepsilon_{r}^{p}}{3}$ et $d \varepsilon_{\text {oct }}^{p}=\frac{\left(d \varepsilon_{p}^{p}-d \varepsilon_{a}^{p}\right)}{3} \sqrt{2}$

avec : $d \varepsilon_{a}^{p}$ : incrément de déformation plastique axiale

et : $\mathrm{d} \varepsilon_{r}^{p}$ : incrément de déformation plastique radiale.

c) Enfin, à l'état ultime défini par le critère Mohr-Coulomb, un palier d'écoúlement plastique est observé.

\section{Définition d'une loi de comportement plastique avec écrouissage à contrainte moyenne cons- tante (Monnet, 1977)}

Le traitement informatique de la formule de Frydman et al. n'est pas immédiat I Aussi faut-il développer une théorie particulière de l'écrouissage du sol qui permette une utilisation simple par la méthode des éléments finis. II n'est pas nécessaire de définir la matrice de rigidité générale par incrément de chargement, comme c'est le cas usuellement. La matrice de rigidité, dans notre théorie, est calculée une fois pour toutes au début du chargement. On calcule les déformations plastiques par un processus itératif (la méthode des contraintes initiales) qui converge rapidement vers la solution théorique. Ceci permet d'économiser de facon considérable le temps de calcul par ordinateur, et donc de rendre l'utilisation du programme plus intéressante.

\subsection{Présentation et hypothèses}

Les quantités $d \varepsilon_{\text {oct }}^{p}$ et $d \varepsilon_{o c t}^{\prime p}$ sont des invariants du tenseur de déformation plastique ; $\sigma_{\text {oct }}$ et $\tau_{\text {oct }}$ sont des invariants du tenseur des contraintes.

Dans la suite, nous considérerons ces quantités comme étant aussi des composantes particulières des vecteurs déformation plastique et contrainte. Ce seront alors des composantes vectorielles orientées dans l'espace (voir sur la figure 1).

On pose :

$\frac{\mathrm{d} \varepsilon_{\text {oct }}^{\mathrm{p}}}{\mathrm{d} \varepsilon_{\text {oct }}^{\mathrm{p}}}=\operatorname{tg} \delta=\frac{1}{K}$

La relation (2) de Frydman se met alors sous la forme :

$f(\sigma, K)=\tau_{\text {oct }}+\sigma_{\text {oct }}\left(\operatorname{tg} \varphi_{\mu}+K\right)=0$

f est fonction de charge puisqu'elle s'annule lorsqu'il y a écoulement plastique. Cette fonction est négative dans le domaine élastique et positive quand on a déséquilibre du sol. D'autre part, la fonction indique un écrouissage puisque sa valeur varie suivant l'état de contrainte du sol, $\mathrm{K}$ étant le paramètre d'écrouissage.

Pour définir parfaitement la déformation plastique, nous admettons avec Nadai (1963), que la distorsion plastique $d \varepsilon^{\prime} \stackrel{p}{p}$ est colinéaire à la contrainte de cisaillement

Fig. 1 Les composantes octaédriques des vecteurs contrainte et déformation plastique

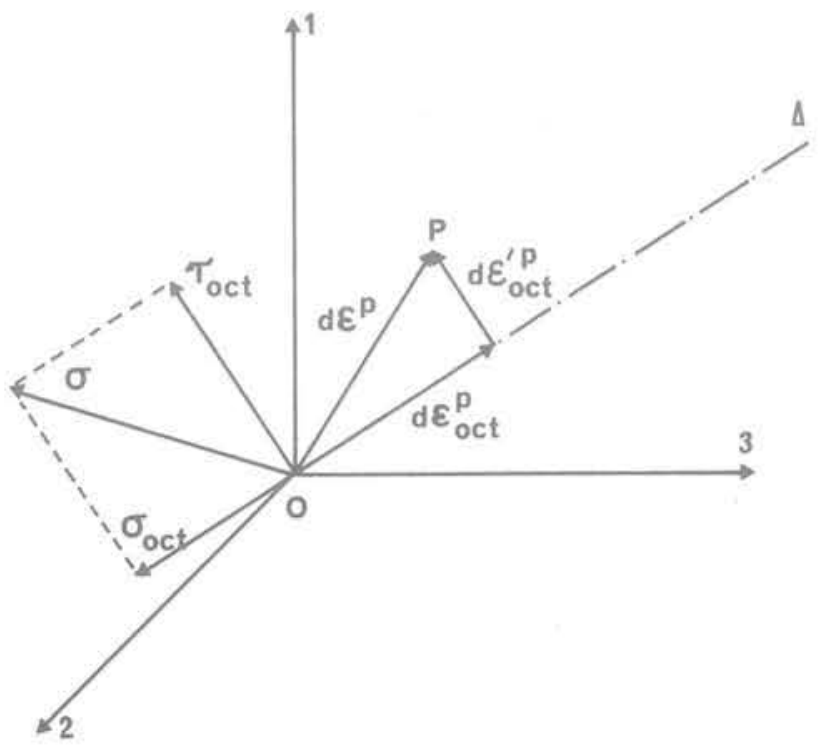


octaédrique :

$\mathrm{d} \varepsilon_{\mathrm{oct}}^{\circ}=\mathrm{d} \lambda \tau_{\text {oct }}$

Les relations (3), (4) et (5) donnent finalement:

$\mathrm{d} \varepsilon_{\text {oct }}^{\rho}=-\mathrm{d} \lambda \tau_{\text {oct }}\left(\frac{\tau_{\text {oct }}}{\sigma_{\text {ort }}}+\operatorname{tg} \varphi_{\mu}\right)$

Nous savons que le vecteur $d \varepsilon^{p}$ est colinéaire à la trissectrice, $d \varepsilon^{\prime} p$ est colinéaire au vecteur cisaillement octaédrique.

\subsection{Changement de repère}

Dans le plan octaédrique perpendiculaire à la trissectrice du trièdre des directions principales, nous définissons un vecteur unitaire $t$ situé à l'intersection de ce plan et du plan contenant l'axe 1 des directions principales et la trissectrice $\Delta$ (voir sur la figure 2 ).

Dans ce même plan octaédrique, nous définissons le vecteur unitaire $\vec{r}$ perpendiculaire à $\vec{t}$ et déduit de celui-ci après une rotation positive de $\frac{\pi}{2}$

Le plan octaédrique contient la composante $\vec{\tau}_{\mathrm{oct}}$ du vecteur contrainte.

L'angle $\gamma$ que fait $\overrightarrow{\tau_{\text {oct }}}$ avec $\vec{t}$ peut se calculer en déterminant d'abord $\vec{\sigma}_{h t}$ et $\vec{\sigma}_{h r}$ (voir figure 2 ). On a les relations :

$\sigma_{h t}=\frac{1}{3 \sqrt{2}}\left(2 \sigma_{1}-\sigma_{2}-\sigma_{3}\right)$

$\sigma_{\mathrm{hr}}=\frac{1}{\sqrt{6}}\left(\sigma_{2}-\sigma_{3}\right)$
D’où il vient :

$\operatorname{tg} \gamma=\frac{\sigma_{\mathrm{hr}}}{\sigma_{\mathrm{ht}}}$

$\operatorname{tg} \gamma=\frac{\sqrt{3}\left(\sigma_{2}-\sigma_{3}\right)}{2 \sigma_{1}-\sigma_{2}-\sigma_{3}}$

Cet angle est défini à $\pi$ près. Pour lever cette indétermination, nous remarquons que $\cos \gamma$ est du signe de $\sigma_{h t}$ puisque $\cos \gamma=\frac{\sigma_{\mathrm{ht}}}{\tau_{\text {oct }}}$ et que $\tau_{\text {oct }}$ est positif par définition. $\left(\sigma_{\mathrm{ht}}\right.$ et $\tau_{\text {oct }}$ sont ici pris comme scalaires) ; $\cos \gamma$ sera alors du signe de $2 \sigma_{1}-\sigma_{2}-\sigma_{3}$.

On effectue un changement de repère qui permet de passer du système local $1^{\cdots}, 2^{\cdots}, 3^{\prime \prime}$ (voir sur la figure 3 ) au système des axes principaux, par trois rotations de valeur $\alpha=45^{\circ}, \beta$ tel que $\cos \left(\frac{\pi}{2}+\beta\right)=\frac{1}{\sqrt{3}}$, et $\gamma$ défini par (7).

Dans le système local, la déformation plastique $\left|d \varepsilon^{D}\right|$ est définie par les coordonnées:

$|x, y, z|^{t}=\left|d \varepsilon_{\text {oct }}^{\circ,} d \varepsilon_{\text {oct }}^{\circ}, 0\right|$

De $(5)$ et $(6)$, il vient:

$|x, y, z|^{\prime}=\left|d \lambda \tau_{o c t},-d \lambda \tau_{o c t}\left(\frac{\tau_{\text {oct }}}{\sigma_{o c t}}+\operatorname{tg} \varphi_{\mu}\right), 0\right|$

Dans le système des axes principaux, les coordonnées de $\left|\mathrm{d} \varepsilon^{p}\right|$ deviennent :

$\left|d \varepsilon^{p}\right|=d \mu|V|=d \mu|a, b, c|^{t}$

avec :

$a=-\frac{\cos \beta \cdot \cos \gamma}{\frac{\tau_{\text {oct }}}{\sigma_{\text {oct }}}+\operatorname{tg} \varphi_{\mu}}+\frac{1}{\sqrt{3}}$

Fig. 2 Définition de la direction du vecteur cisaillement octaédrique par l'angle $\gamma$

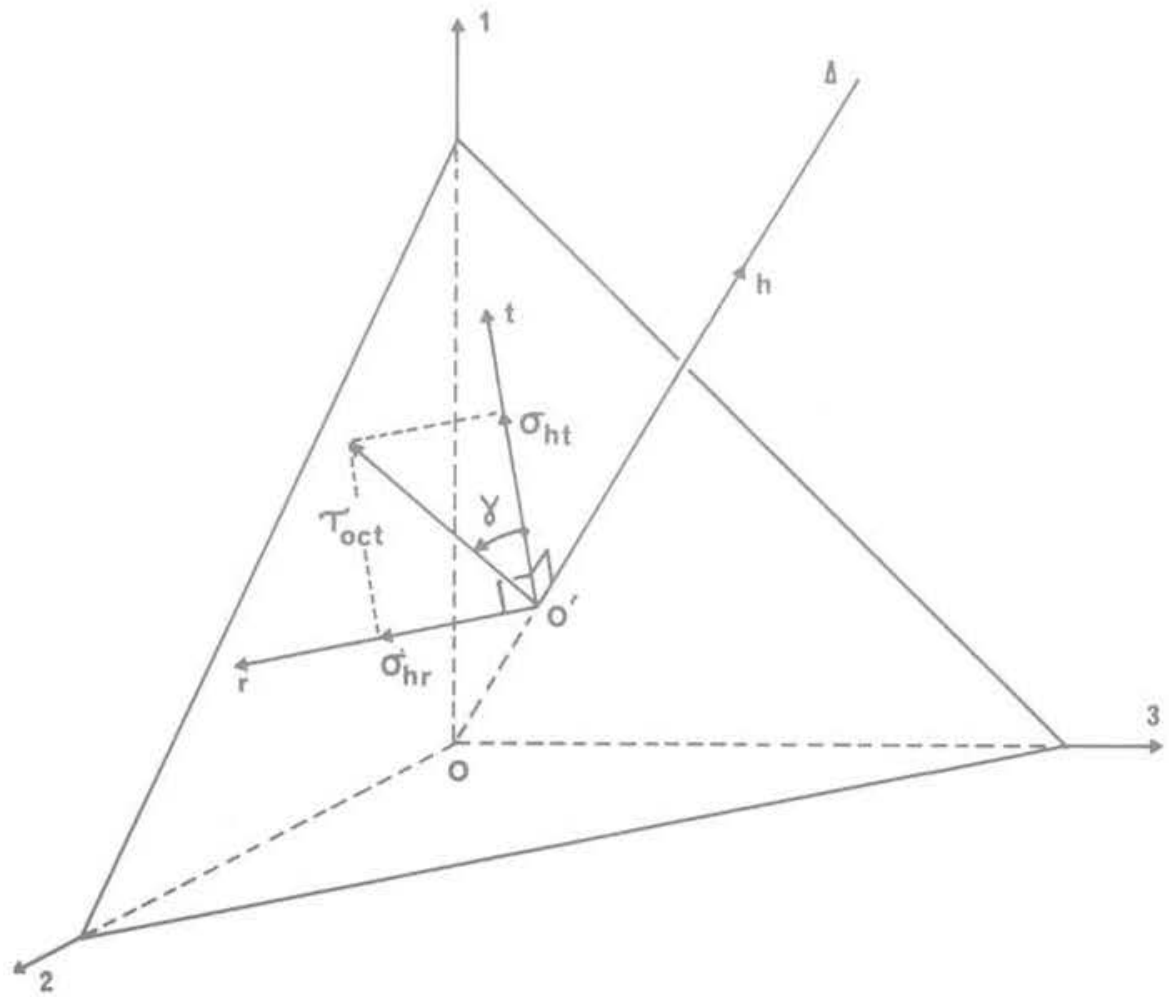




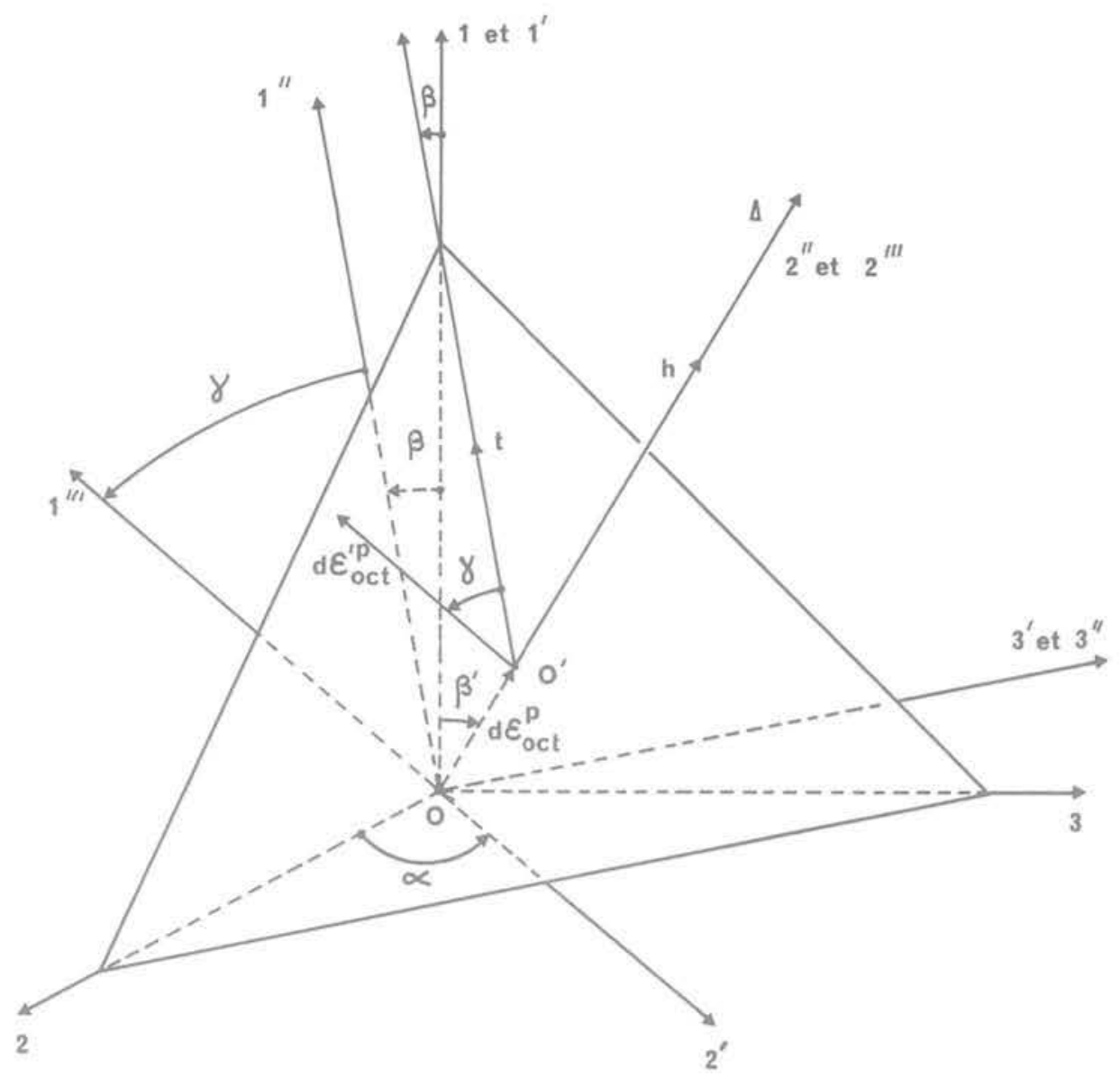

Fig. 3 Définition du changement de repère par les trois angles : $\alpha, \beta$ et $\gamma$

$\mathrm{b}=-\frac{\cos \alpha \cdot \sin \beta \cdot \cos \gamma+\sin \alpha \cdot \sin \gamma}{\frac{\tau_{\text {oct }}}{\sigma_{\text {oct }}}+\operatorname{tg} \varphi_{\mu}}+\frac{1}{\sqrt{3}}$

$c=-\frac{\cos \alpha \cdot \sin \beta \cdot \cos \gamma-\sin \alpha \cdot \sin \gamma}{\frac{\tau_{\text {oct }}}{\sigma_{\text {oct }}}+\operatorname{tg} \varphi_{\mu}}+\frac{1}{\sqrt{3}}$

et : $\quad d \mu=\frac{-d \lambda}{\tau_{\text {oct }}\left(\frac{\tau_{\text {oct }}}{\sigma_{\text {oct }}}+\operatorname{tg} \varphi_{\mu}\right)}$

$\mathrm{d} \mu$ est un scalaire d̀ déterminer.

\subsection{Résolution}

Nous effectuons une résolution classique de l'élastoplasticité oủ :

$f(\sigma, K)=\tau_{\text {oct }}+\sigma_{\text {oct }}\left(\operatorname{tg} \varphi_{\mu}+K\right)$

est la fonction de charge, et oú :

$|d \varepsilon p|=d \mu|V|$

remplace l'hypothèse de la normalité par un potentiel non associé (cas du matériau " non standard»). Le matériau "standard » obéit au principe du travail plastique maximum $|\mathrm{d} \varepsilon p|=\mathrm{d} \lambda\left\langle\frac{\partial f}{\partial \sigma}\right|$. Cette relation est appelée aussi loi de normalité puisqu'elle impose que le vecteur incrément de déformation plastique d $\varepsilon^{\mathrm{p}}$ soit perpendiculaire à la surface géométrique définie par la fonction $f$. Pour le matériau " non standard», et pour les sols en général, la loi de nor- malité n'est pas applicable. On remplace alors la fonction $\left\lfloor\frac{\partial f}{\partial \sigma}\right\rfloor$ par $\left\{\frac{\partial g}{\partial \sigma}\right\} . g$ est appelé : potentiel plastique non associé,

Nous calculons la valeur de la fonction $f$ pour tous les points du modèle d'éléments finis. Quand la fonction de charge est positive, nous effectuons le rééquilibrage en calculant les variations de contraintes $\{\delta \sigma\}$ telles que :

$\left.|\delta \sigma|^{t}=-\mathrm{d} \lambda *|\mathrm{~V}| \mathrm{TE}\right]$

avec :

$$
d \lambda^{*}=\frac{f\left(\sigma_{\gamma}, K\right)}{|V|^{2}\lfloor E]\left\{\frac{\partial f}{\partial \sigma}\right\}-|V|^{*}\left\{\frac{\partial K}{\partial\left(d \varepsilon^{p}\right)}\right\} \frac{\partial f}{\partial K}}
$$

[E] est la matrice de la loi de Hooke généralisée. Nous pouvons remarquer que la fonction de charge proposée correspond à un critère de Drucker :

$f=\alpha J_{1}+J_{2}^{1 / 2}$

avec : $J_{1}=\sigma_{1}+\sigma_{2}=\sigma_{3}=3 \sigma_{\text {oct }}$

$J_{2}=\frac{1}{6}\left[\left(\sigma_{1}-\sigma_{2}\right)^{2}+\left(\sigma_{2}-\sigma_{3}\right)^{2}+\left(\sigma_{3}-\sigma_{1}\right)^{2}\right]=\frac{3}{2} \tau_{\text {oct }}^{2}$

$\alpha=\frac{1}{\sqrt{6}}\left(\operatorname{tg} \varphi_{\mu}+K\right)$

et avec l'hypothèse du matériau "non standard ». 


\section{Etude du comportement du sol à l'état ultime}

Bent et Hansen (1968) puis Radenkovic (1961) ont proposé un comportement "non standard" du sol à l'état ultime. Pour eux, le vecteur incrément de déformation plastique n'est pas perpendiculaire à la surface de charge, mais il est décalé. Nous avons cherché à définir quantitativement cet écart. Des essais triaxiaux drainés réalisés sur des sables (Darmar et Plessiet - 1964) et sur des billes de plomb (Newland et Allely - 1957) ont donc été analysés dans ce sens.

Pendant l'essai triaxial de révolution, les contraintes principales $\sigma_{2}$ et $\sigma_{3}$ sont identiques. Si l'on représente l'état de contrainte dans lespace des axes principaux, les points représentatifs de l'état de charge sont situés dans le plan contenant l'axe 1 et la bissectrice des axes 2 et 3 .

Nous avons représenté sur les figures $4,5,6$ et 7 les points caractérisant la rupture de l'échantillon dans ce plan particulier. Nous constatons que tous les points sont alignés. Si nous essayons de faire passer une droite de régression par ces points et par l'origine, nous obtenons des coefficients de corrélation très proches de 1 (voir sur le tableau 8 , les colonnes 3,4 et 5).

Nous avons porté également sur ces graphes, les vecteurs incréments de déformation plastique à la rupture. Si le matériau était "standard\%, ces vecteurs devraient être perpendiculaires à la ligne de rupture représentée par la droite en trait plein sur les figures. Nous voyons que ce n'est pas le cas. Les matériaux testés (échantillon de billes de plomb pour Newland et Allely, et sable drainé pour Darmar et Plessiet) se comportent de façon «non standard ".

Partant de l'origine, on abaisse la perpendiculaire aux vecteurs incrément de déformation plastique. Nous obtenons une seconde série de points dont les coordonnées sont portées en colonnes 7 et 8 sur le tableau 8 . En calculant la droite de régression passant par tous ces points et par l'origine, nous trouvons un coefficient de corrélation très proche de 1 (voir la colonne 9 sur le tableau 8). Ceci indique qu'à la rupture, le vecteur incrément de déformation plastique est perpendiculaire à une surface de charge particulière, représentée ici par les droites en trait interrompu. Cette surface de charge peut être considérée comme le passage d'une surface de charge de Mohr Coulomb dont l'angle $\varphi^{\prime \prime}$ (voir colonne 10 sur le tableau 8 ) est inférieur à la valeur $\varphi$ (voir la colonne 6 sur le tableau 8) du frottement du sol à la rupture, comme le propose Salençon (1974).

Dans la programmation, nous considérons qu'à l'état ultime, lorsque le critère de Mohr-Coulomb du matériau pulvérulent :

$f(\sigma)=\sigma_{\max }-\sigma_{\min }+\sin \varphi\left(\sigma_{\max }+\sigma_{\min }\right)$

devient positif ou nul, il se produit un écoulement plastique "non standard" défini par:

$\left|d \varepsilon^{p}\right|=\left\{\begin{array}{c}d \varepsilon_{\text {max }}^{p} \\ d \varepsilon_{\min }^{p} \\ d \varepsilon_{\text {moy }}^{p}\end{array}\right\}=d \lambda\left[\begin{array}{c}\sin \varphi^{\prime \prime}+1 \\ \sin \varphi^{\prime \prime}-1 \\ 0\end{array}\right]$

Les variations de contraintes sont calculées par:

$\left\langle\left.\mathrm{d} \sigma\right|^{\mathrm{t}}=-\left|\mathrm{d} \varepsilon^{\mathrm{P}}\right|^{\mathrm{t}}[\mathrm{E}]\right.$

et nous appliquons la méthode des contraintes initiales.

Les calculs de 13 essais triaxiaux de révolution, que nous développons dans la suite de cet article, indiquent que l'on a la relation suivante:

$\varphi-\varphi^{\prime \prime}=\varphi_{\mu} \quad$ (11) (voir les figures 22 et 23).

La connaissance de $\varphi$ et $\varphi_{\mu}$ permet donc de définir $\varphi^{*}$. ( $\varphi$ et $\varphi_{\mu}$ seront des données du programme de calcul).

Fig. 8 Tableau récapitulatif des résultats définissant la rupture et la direction du vecteur déformation plastique

\begin{tabular}{|c|c|c|c|c|c|c|c|c|c|}
\hline 1 & 2 & 3 & 4 & 5 & 6 & 7) & 8 & 9 & 10 \\
\hline Matériau & $\begin{array}{c}\text { Essai } \\
\text { numéro }\end{array}$ & $\begin{array}{l}\text { Pression } \\
\text { axiale } \\
\text { (bar) }\end{array}$ & $\begin{array}{l}\text { Pression } \\
\text { radiale } \\
\text { (bar) }\end{array}$ & $\begin{array}{c}\text { Coefficient } \\
\text { de } \\
\text { corrélation }\end{array}$ & $\stackrel{\varphi}{\text { (degré) }}$ & $\begin{array}{c}\text { Coordonnée } \\
\text { axiale }\end{array}$ & $\begin{array}{c}\text { Coordonnée } \\
\text { radiale }\end{array}$ & $\begin{array}{c}\text { Coefficient } \\
\text { de } \\
\text { corrélation }\end{array}$ & $\begin{array}{c}\varphi^{\prime \prime} \\
\text { (degré) }\end{array}$ \\
\hline $\begin{array}{l}\text { Billes de } \\
\text { plomb } \\
\text { lubrifiées } \\
n=39 \%\end{array}$ & $\begin{array}{l}13 \\
14\end{array}$ & $\begin{array}{c}13,22 \\
6,34 \\
0,0\end{array}$ & $\begin{array}{l}5,52 \\
2,76 \\
0,0\end{array}$ & 0,9997 & 24,2 & $\begin{array}{l}8,7 \\
4,27 \\
0,0\end{array}$ & $\begin{array}{l}7,95 \\
3,82 \\
0,0\end{array}$ & 0,9999 & 2.6 \\
\hline $\begin{array}{l}\text { Billes de } \\
\text { plomb } \\
n=43 \%\end{array}$ & $\begin{array}{l}46 \\
52 \\
53 \\
44\end{array}$ & $\begin{array}{c}22,84 \\
10,21 \\
4,89 \\
1,65 \\
0,0\end{array}$ & $\begin{array}{l}5,52 \\
2,76 \\
1,38 \\
0,52 \\
0,0\end{array}$ & 0,9985 & 37,1 & $\begin{array}{r}13,95 \\
6,90 \\
3,45 \\
1,20 \\
0,0\end{array}$ & $\begin{array}{c}11,03 \\
4,98 \\
2,33 \\
0,85 \\
0,0\end{array}$ & 0,9989 & 7,2 \\
\hline $\begin{array}{l}\text { Billes de } \\
\text { plomb } \\
n=39 \%\end{array}$ & $\begin{array}{r}19 \\
25 \\
4 \\
40\end{array}$ & $\begin{array}{c}26,77 \\
12,42 \\
5,11 \\
2,18 \\
0,0\end{array}$ & $\begin{array}{l}5,52 \\
2,76 \\
1,38 \\
0,52 \\
0,0\end{array}$ & 0,9982 & $40: 7$ & $\begin{array}{c}17,10 \\
7,65 \\
3,75 \\
1,57 \\
0,0\end{array}$ & $\begin{array}{c}11,14 \\
5,30 \\
2,17 \\
0,90 \\
0,0\end{array}$ & 0,9993 & 12.0 \\
\hline Sable & $\begin{array}{l}1 \\
2 \\
3\end{array}$ & $\begin{array}{c}12,54 \\
8,76 \\
4,38 \\
0,0\end{array}$ & $\begin{array}{l}2,94 \\
1,96 \\
0,98 \\
0,0\end{array}$ & 0,9997 & 38.7 & $\begin{array}{l}7,85 \\
5,70 \\
3,00 \\
0,0\end{array}$ & $\begin{array}{l}6,01 \\
4,10 \\
2,01 \\
0,0\end{array}$ & 0,9989 & 8,3 \\
\hline
\end{tabular}




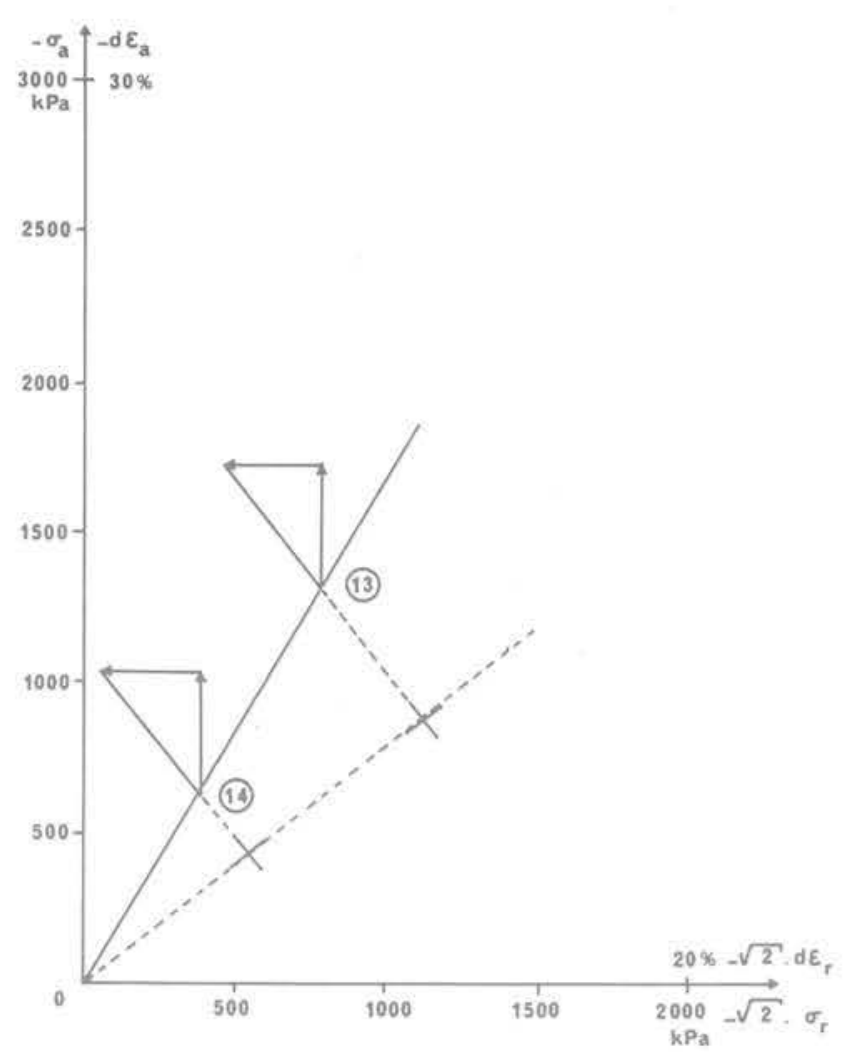

Fig. 4 Les points caractérisant les contraintes et les vecteurs incrément de déformation plastique à la rupture pour les essais 13 et 14

Fig. 6 Les points caractérisant les contraintes et les vecteurs incrément de déformation plastique à la rupture pour les essais $19,25,4$ et 40

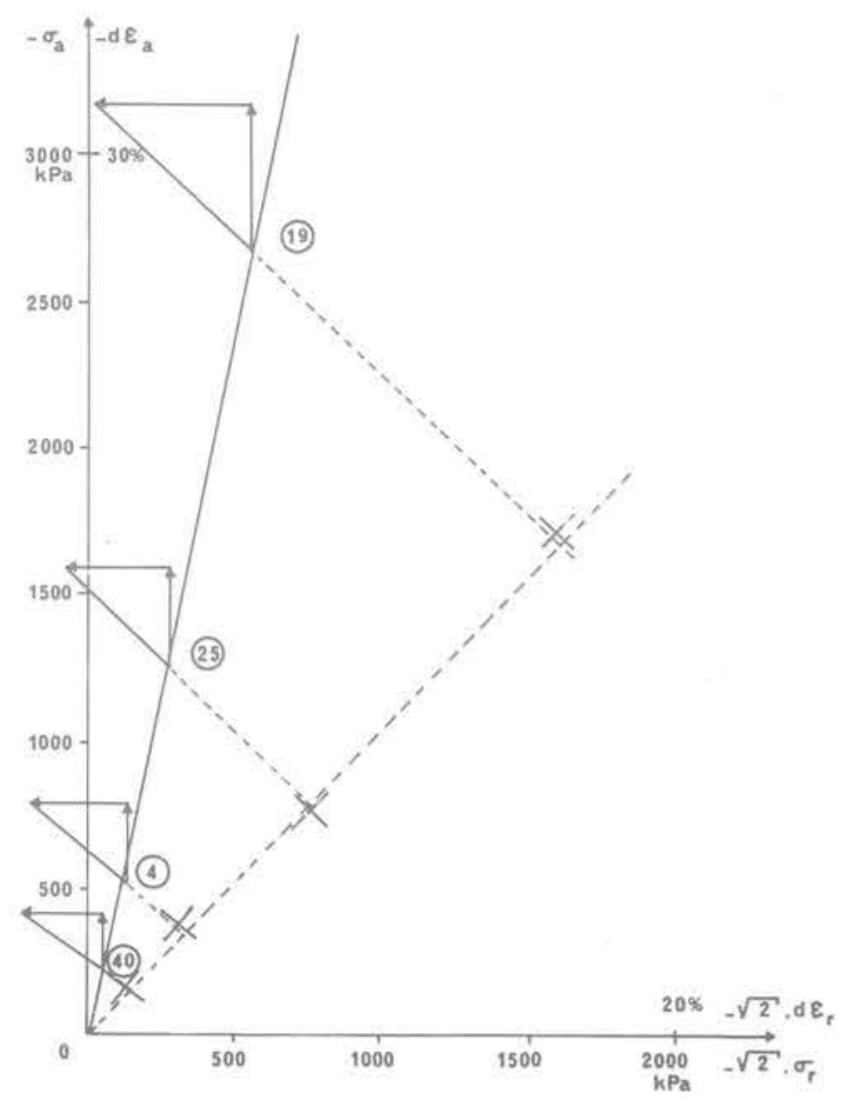

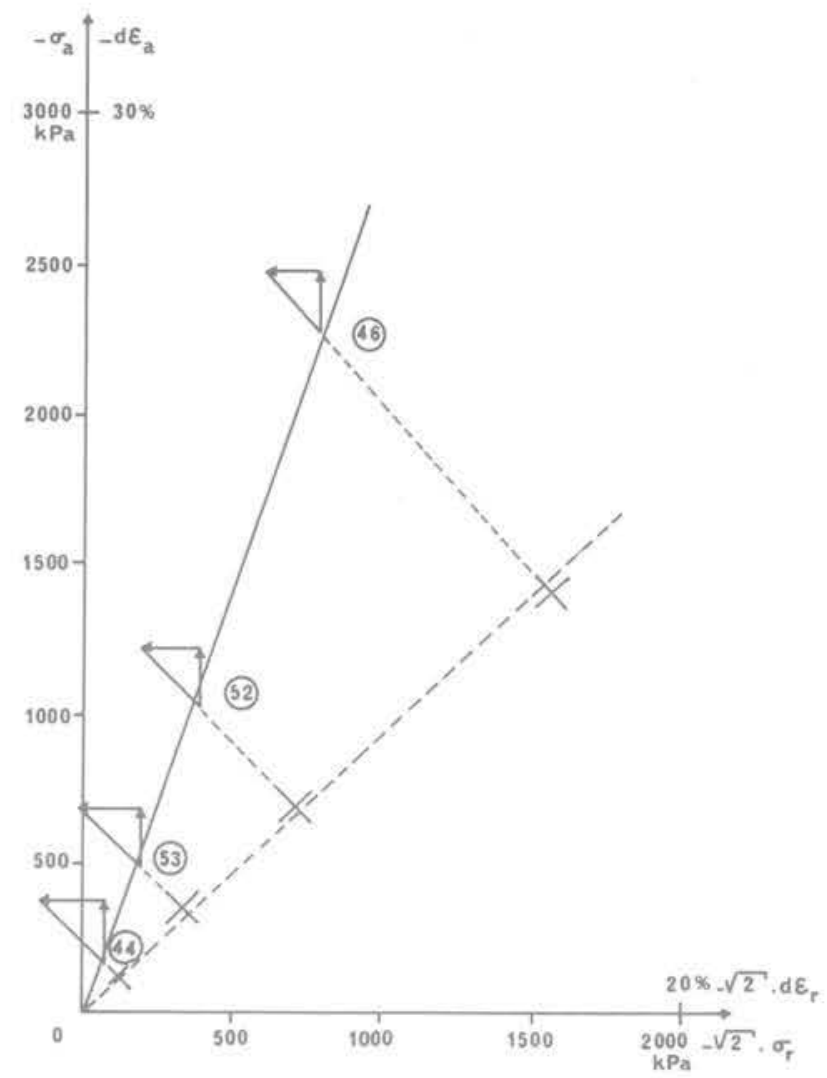

Fig. 5 Les points caractérisant les contraintes et les vecteurs incrément de déformation plastique à la rupture pour les essais $46,52,53,44$

Fig. 7 Les points caractérisant les contraintes et les vecteurs incrément de déformation plastique à la rupture pour les essais 1, 2 et 3

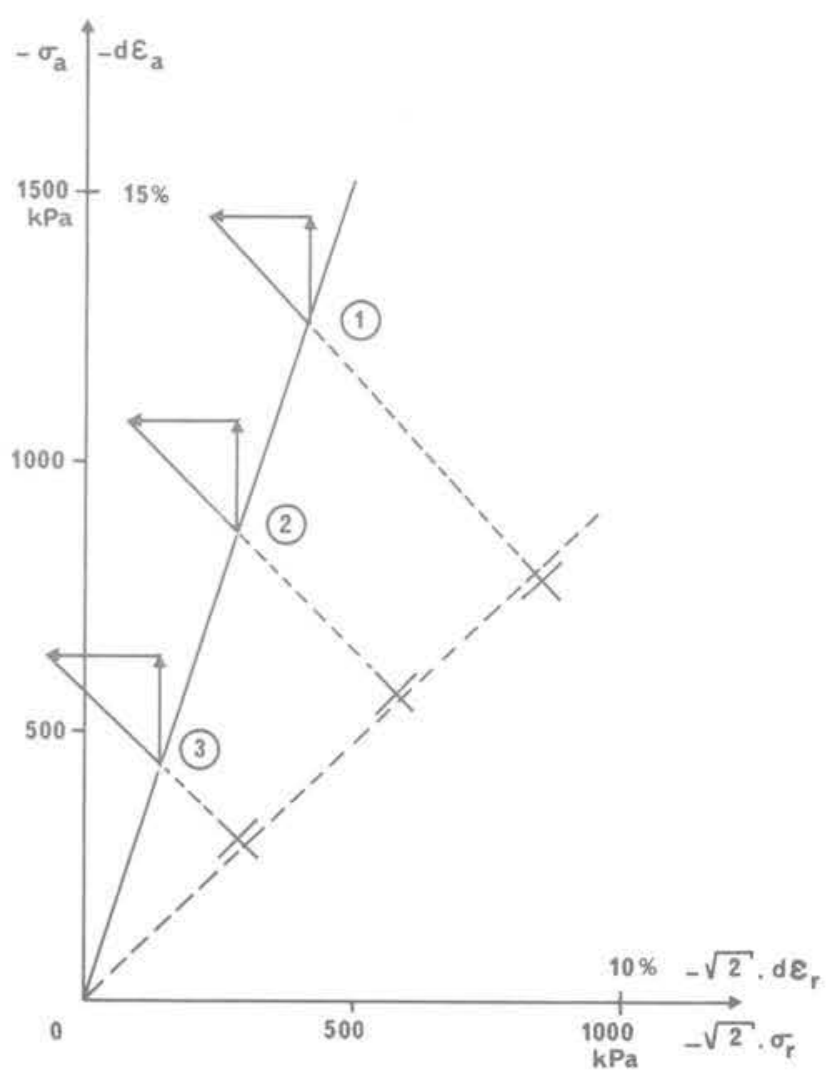




\section{Calcul de 13 essais triaxiaux de révolution}

La théorie de l'écrouissage à contrainte moyenne constante et de la plasticité "non standard » étant élaborée. nous nous sommes proposés de comparer les résultats de calcul et ceux de l'expérimentation dans le cas d'essais triaxiaux. Nous avons calculé plusieurs séries d'essais de façon à ce que les quatre paramètres de la loi de comportement varient. Au cours de l'essai triaxial, la contrainte moyenne n'est pas constante, mais nous allons voir que la théorie peut être appliquée à cet essai sans donner des écarts significatifs par rapport à l'expérience.

\subsection{Choix des paramètres}

La loi de comportement peut être schématisée comme sur la figure 9.

Dans le domaine des faibles sollicitations, le sol se comporte élastiquement. La valeur du module de Young $E$ est déduite de la pente initiale de la courbe déviateur en fonction de l'écrasement; la valeur du coefficient de Poisson $\mathrm{v}$. est déduite de la pente initiale de la courbe variation de volume en fonction de l'écrasement.

Pour l'écrouissage, le seul paramètre à définir est l'angle de frottement grain sur grain $\varphi_{\mu}$. Cet angle est constant pour un matériau donné.

Enfin, au moment de résistance maximum du sol, nous considérons qu'il se produit un écoulement plastique «non standard "). L'angle $\varphi$ de frottement ultime est défini par la droite de Mohr-Coulomb. L'angle $\varphi_{\mu}$ étant supposé connu, la relation (11) donne la valeur $\varphi^{\prime \prime}$ définissant le potentiel non associé.

\subsection{Essais de Darmar et Plessiet}

Les essais sont réalisés à l'appareil triaxial de révolution. sur un sable de densité $14,6 \mathrm{kN} / \mathrm{m}^{3}$ environ. Les extrémités de l'éprouvette frottent sur l'embase et la tête de compression. L'échantillon est saturé et drainé, ce qui permet de mesurer les variations de volume. Les essais sont réalisés sous trois étreintes latérales: $98 \mathrm{kPa}, 196 \mathrm{kPa}$ et $294 \mathrm{kPa}$ (Monnet, 1977).

La figure 10 représente le maillage utilisé (constitué d'éléments à trois nœuds) pour le calcul de l'essai triaxial. Seul $1 / 4$ de l'échantillon a été représenté. II est limité à gauche par l'axe de symétrie de révolution et à la partie inférieure, par un plan de symétrie, La partie supérieure du maillage correspond à la tête de compression qui est en duralumin. Nous avons pris comme caractéristiques pour ces éléments : $E=90300000 \mathrm{kPa}$ et $v=0,33$. II n'y a pas d'éléments de continuité entre le sol et la tête de compression puisque l'essai a eu lieu sans lubrification entre ces parties.

Au premier pas de chargement, nous appliquons l'étreinte triaxiale choisie sur tout le bord droit du modèle et sur tout le bord supérieur.

Ensuite, nous chargeons uniquement la partie fléchée sur la figure 10. Cette zone correspond à l'emplacement du piston qui vient appuyer sur la tête de compression.

\section{Résultats :}

Comparaison des différentes grandeurs calculées et mesurées.

\section{Courbes déviateur en fonction de l'écrasement}

Nous avons comparé sur la figure 11 les résultats calculés (en noir) et expérimentaux (en clair) pour les trois valeurs de l'étreinte latérale. La coïncidence est bonne en tout point des courbes. Nous observons enfin que le dernier point calculé pour chaque étreinte correspond à la rupture de l'échantillon puisque la zone en équilibre limite de Mohr-Coulomb couvre toute la partie centrale (voir sur la figure 13 pour l'essai 1 ). Cette valeur calculée de la rupture correspond assez bien à la valeur expérimentale.

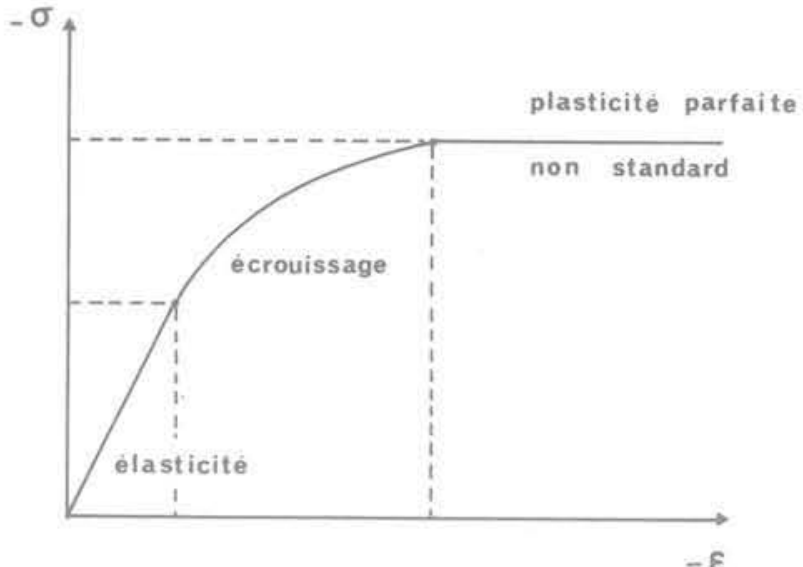

Fig. 9 Schéma de la loi de comportement utilisée dans le programme d'éléments finis

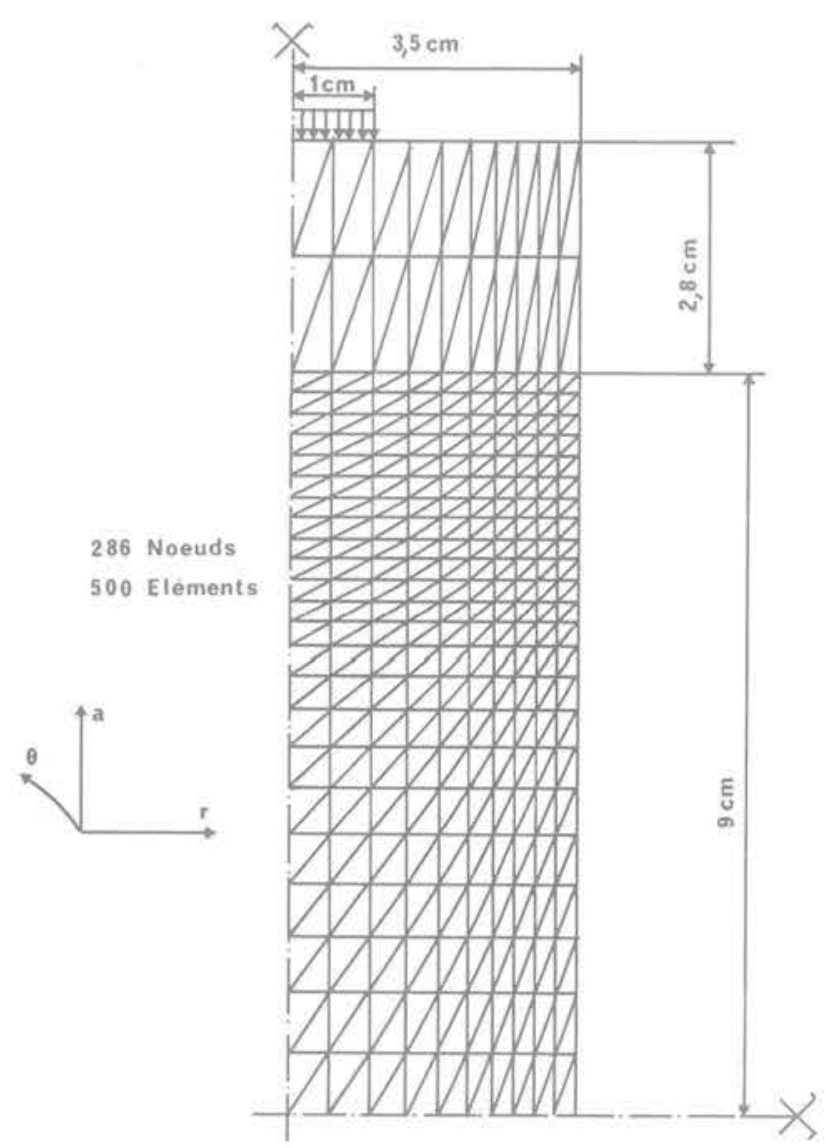

Fig. 10 Maillage de calcul de l'essai triaxial de révolution, pour les essais 1,2 et 3

Courbes variation de volume en fonction de l'écrasement La figure 12 représentè la comparaison des variations relatives de volume en fonction du raccourcissement de l'échantillon. La coïncidence est bonne, compte tenu de la grande échelle verticale utilisée. Les courbes calculées des essais 2 et 3 sont presque confondues.

\section{Etendue des zones plastiques au cours de l'essai}

II s’agit encore de l'essai 1 à $294 \mathrm{kPa}$ porté sur la figure 13. Au déviateur $500 \mathrm{kPa}$ tout le sol est en élasticité. $\mathrm{A}$ $600 \mathrm{kPa}$, brusquement apparaît au centre de l'échantillon une zone d'écrouissage qui remonte progressivement jusque sous la tête de compression. Une petite zone en équilibre de Mohr-Coulomb apparaît au pourtour de l'échantillon à $3,5 \mathrm{~cm}$ environ au-dessous de la tête de compression. Cette zone gagne rapidement du volume jusqu'à occuper tout le centre du cylindre de sol à la rupture obtenue pour un déviateur de $1000 \mathrm{kPa}$. 


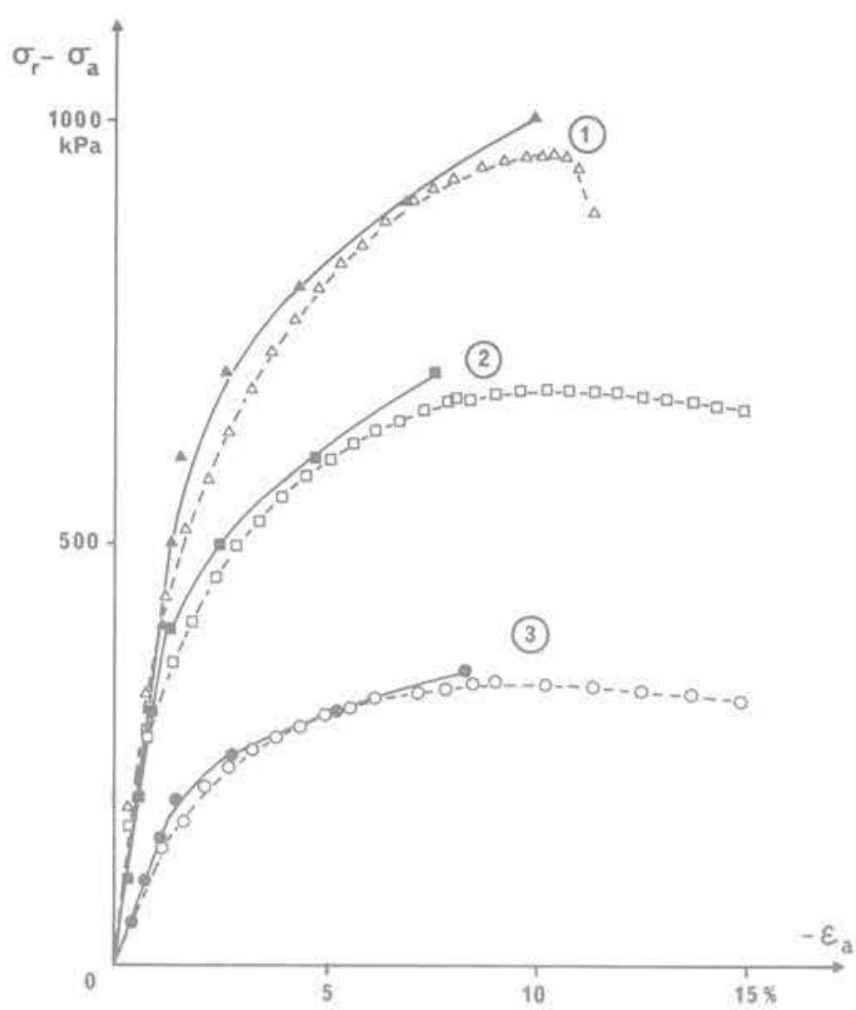

Fig. 11 Courbe déviateur des contraintes-tassement, pour les essais 1, 2 et 3 sur un sable - Comparaison des résultats expérimentaux (en clair) et calculés (en noir) pour trois valeurs de l'étreinte latérale

Fig. 13 Etat de l'échantillon pour chaque valeur du déviateur calculé à l'essai 1 à $294 \mathrm{KPa}$

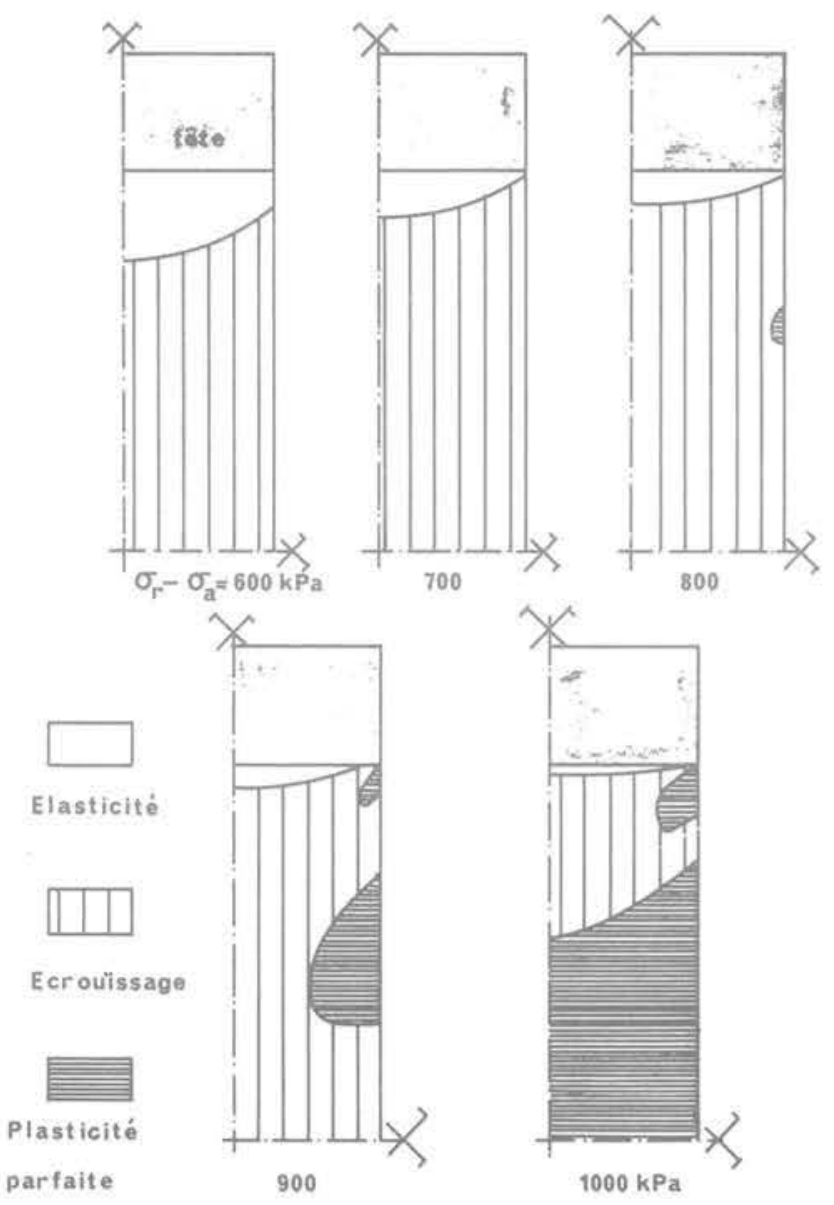

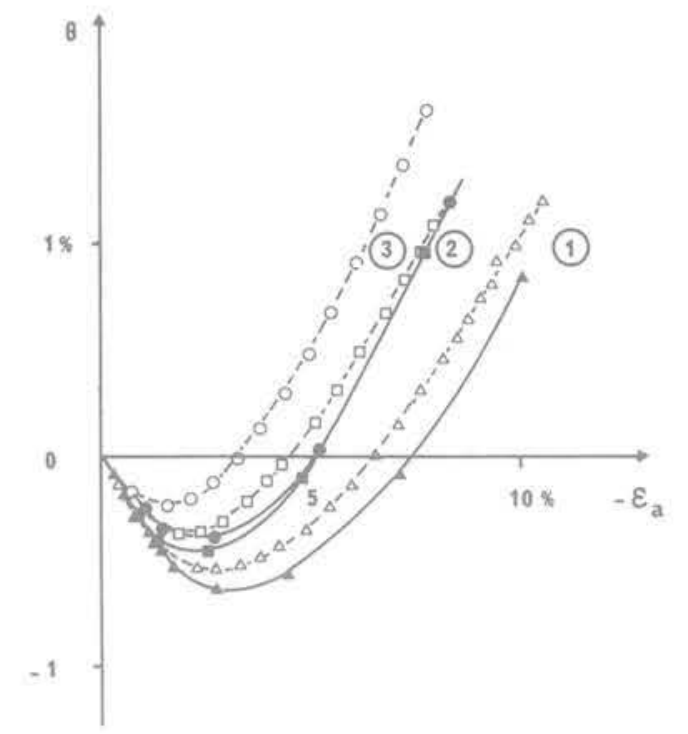

Fig. 12 Courbes variation de volume-tassement, pour les essais 1, 2 et 3 sur un sable - Comparaison des résultats expérimentaux (en clair) et calculés (en noir) pour trois valeurs de l'étreinte latérale

Fig. 14 Lignes d'égale contrainte verticale, pour une contrainte imposée de $-1200 \mathrm{kPa}$ (déviateur $900 \mathrm{kPa}$ ), et pour l'essai 1 à $294 \mathrm{kPa}$

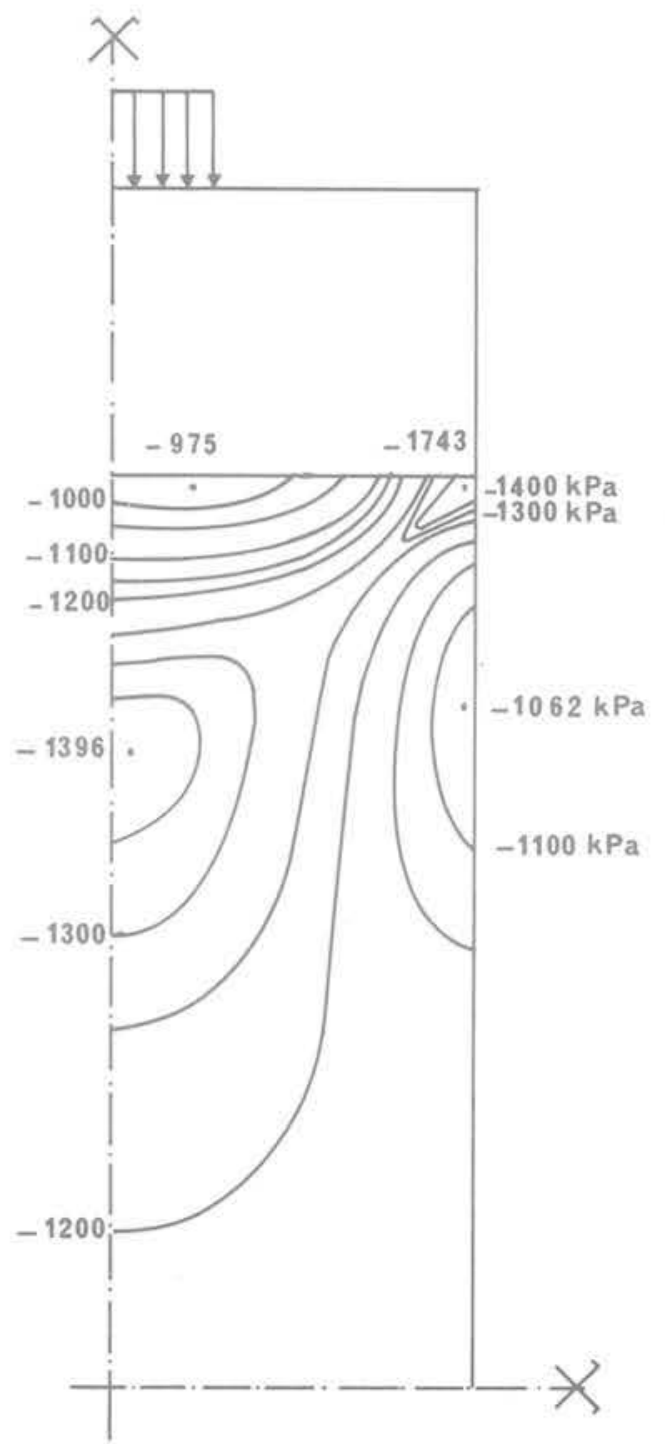


Courbes d'égale contrainte verticale pour le déviateur $900 \mathrm{kPa}$

La figure 14 représente les résultats de l'essai 1 à $294 \mathrm{kPa}$. La contrainte verticale moyenne pour ce déviateur est de $-1200 \mathrm{kPa}$.

Nous observons le long de laxe de symétrie, tout d'abord une contrainte $20 \%$ plus faible que la contrainte moyenne, puis en descendant une valeur $15 \%$ plus forte. $\mathrm{Si}$ nous regardons ce qui se passe à la périphérie, nous observons sous la tête de compression une contrainte $45 \%$ plus forte que la valeur moyenne, puis en redescendant une valeur $10 \%$ plus faible.

Nous remarquons également qu'une section horizontale de l'échantillon à quel niveau que ce soit donne au total une charge verticale moyenne constante.

Les résultats sous la tête de compression sont analogues à ce qui se passe sous une fondation rigide : les contraintes sont maximum au bord et minimum au centre.

\subsection{Essais de Newland et Allely}

La méthode de calcul a été adaptée au programme ROSALIE (Guellec - 1976) du Laboratoire Central des Ponts et Chaussées, ce qui a permis de calculer ces essais. Le rééquilibrage est limité à 20 itérations. Le maillage utilisé est présenté sur la figure 15. Il s'agit de quadrilatères à huit nœuds. Le modèle présente une symétrie axiale par rapport au bord gauche et un plan de symétrie par rapport au bord inférieur. Les éléments de continuité placés entre le piston et le matériau transmettent uniquement les déplacements verticaux. Les déplacements horizontaux sont libres. Le matériau testé est un échantillon de billes de plomb.

Fig. 15 Maillage de calcul des essais triaxiaux de révolu. tion réalisés sur les billes de plomb

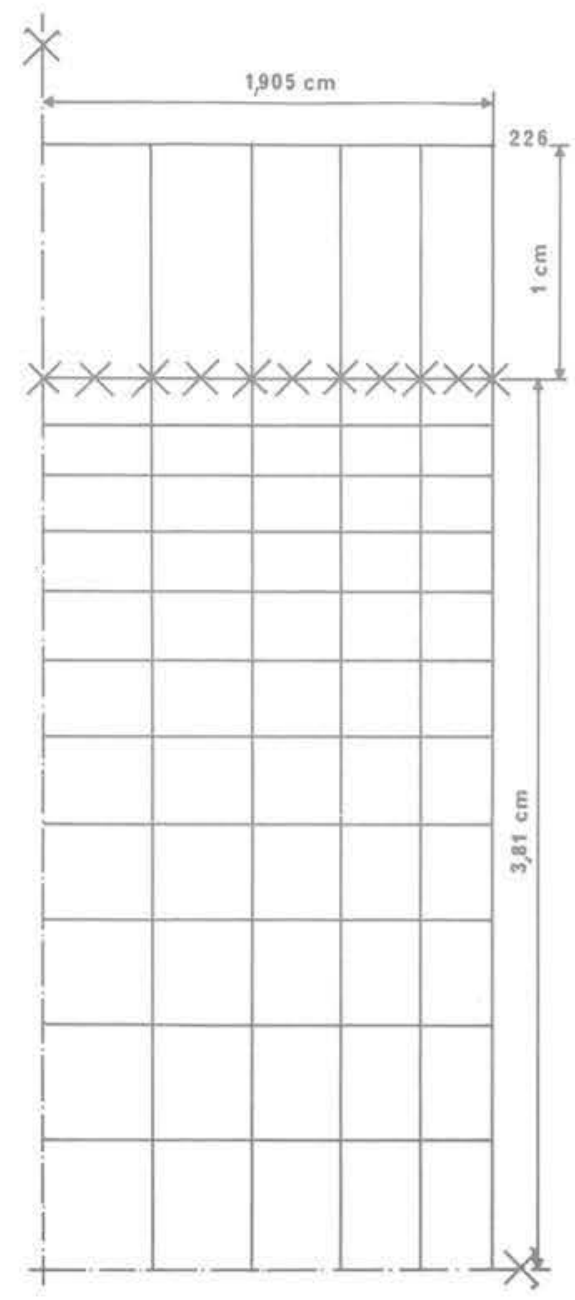

Essai du matériau à la porosité de $39 \%$

Les calculs effectués pour les quatre étreintes latérales sont portés en noir, et les résultats expérimentaux en clair. On voit sur la figure 16, le déviateur en fonction de l'écrasement. Les derniers points calculés correspondent tous à la rupture de la totalité de l'échantillon. Le passage de l'écrouissage à la rupture s'effectue toujours brusquement pour tout le cylindre de matériau. En effet, les contraintes calculées sont égales en tout point à l'échantillon. Ceci montre bien l'importance que joue la lubrification des extrémités du cylindre de sol. Nous observons que pour les étreintes latérales testées $(52 \mathrm{kPa}, 138 \mathrm{kPa}, 276 \mathrm{kPa}$ et $552 \mathrm{kPa}$ ), la coïncidence est bonne entre l'expérience et le calcul par éléments finis. Seul l'essai 4 diverge un peu, mais ceci est dû à l'expérience puisque l'échantillon a cédé plus tôt que ce que prévoit le critère de Mohr-Coulomb.

Nous voyons sur la figure 17 , la variation de volume en fonction de l'écrasement. La coïncidence est très bonne entre les courbes.

\section{Essai du matériau lubrifié à la porosité de $39 \%$}

L'angle $\varphi_{\mu}$ est plus faible que pour les essais précédents. Pour les deux étreintes latérales testées $(552 \mathrm{kPa}$ et $276 \mathrm{kPa}$ ) nous voyons sur les figures 18 et 19 que les résultats coîncident très bien avec l'expérience.

Essai du matériau à la porosité de $43 \%$

L'angle $\varphi_{\mu}$ est le même que pour les premiers essais. Nous voyons que pour les quatre étreintes latérales $(552 \mathrm{kPa}$, $276 \mathrm{kPa}, 138 \mathrm{kPa}$ et $52 \mathrm{kPa}$ ), la coïncidence est très bonne entre les deux séries de courbes (figures 20 et 21).

Fig. 16 Courbes déviateur des contraintes-tassement, pour les essais 19, 25, 4 et 40 sur des billes de plomb. Comparaison des résultats calculés (en noir) et des résultats expérimentaux (en clair)

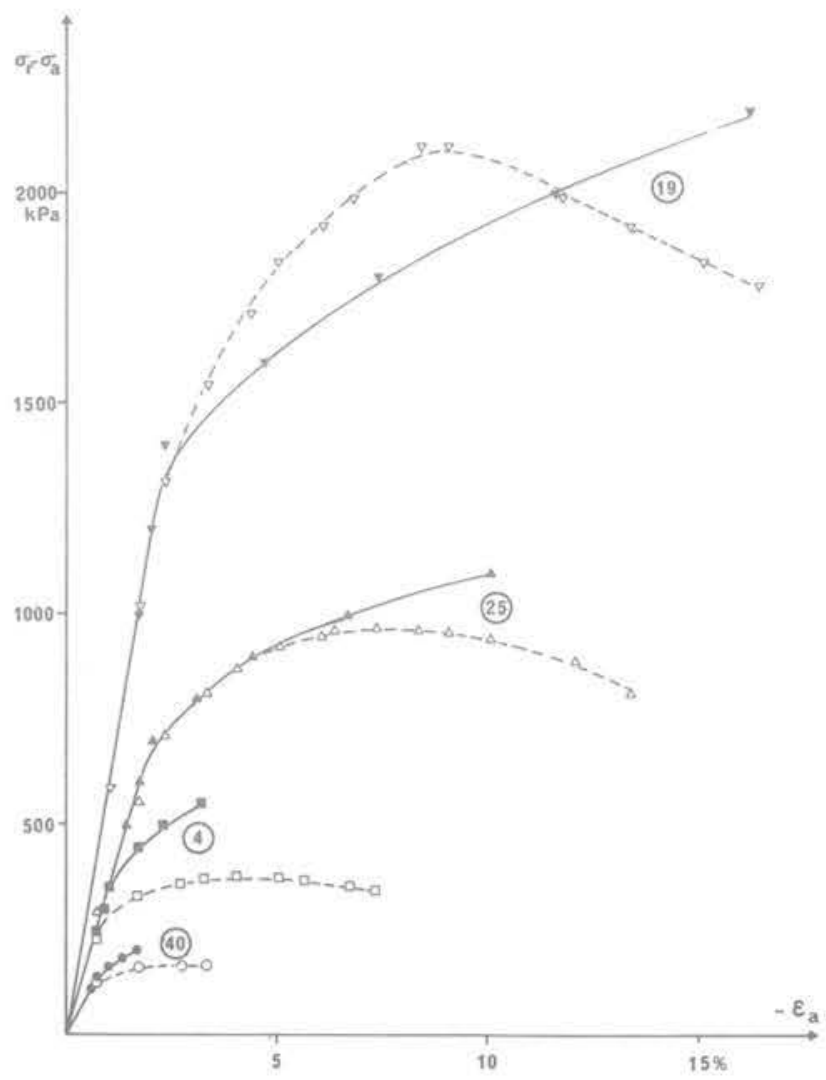




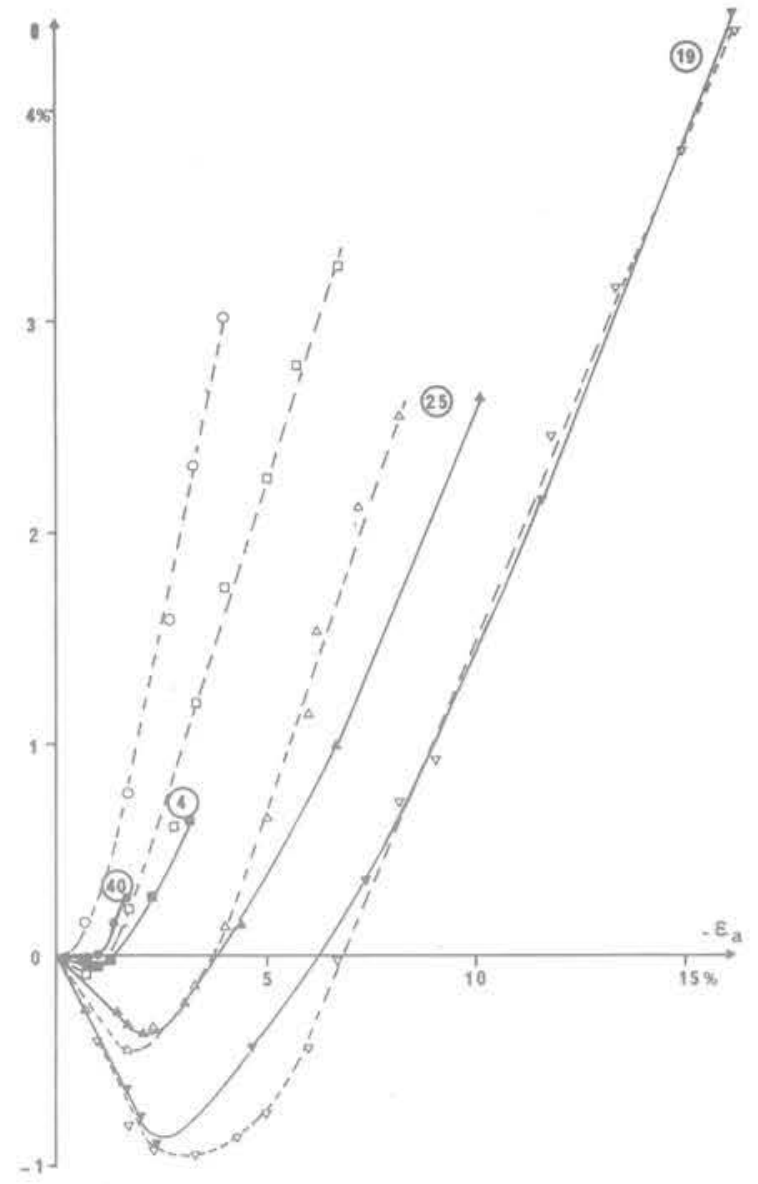

Fig. 17 Courbes variation de volume-tassement pour les essais 19, 25, 4 et 40 sur des billes de plomb. Comparaison des résultats calculés (en noir) et des résultats expérimentaux (en clair)

Fig. 20 Courbes déviateur des contraintes-tassement, pour les essais 46, 52, 53 et 44 sur des billes de plomb. Comparaison des résultats calculés (en noir) et des ré. sultats expérimentaux (en clair)

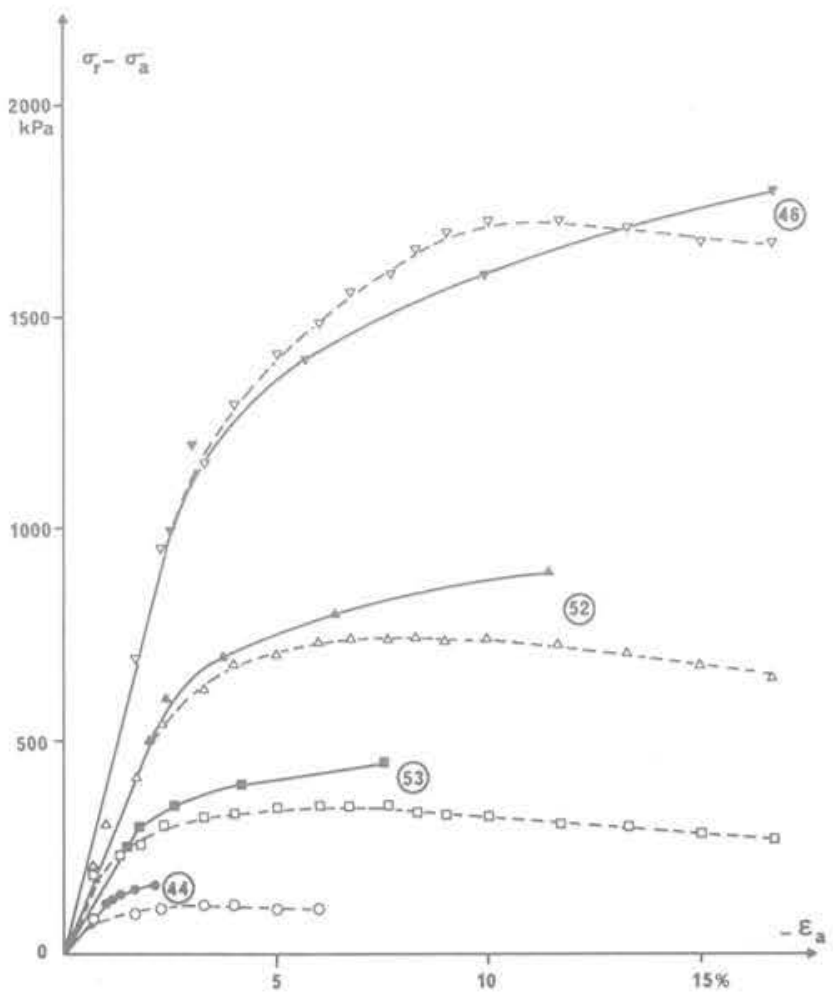

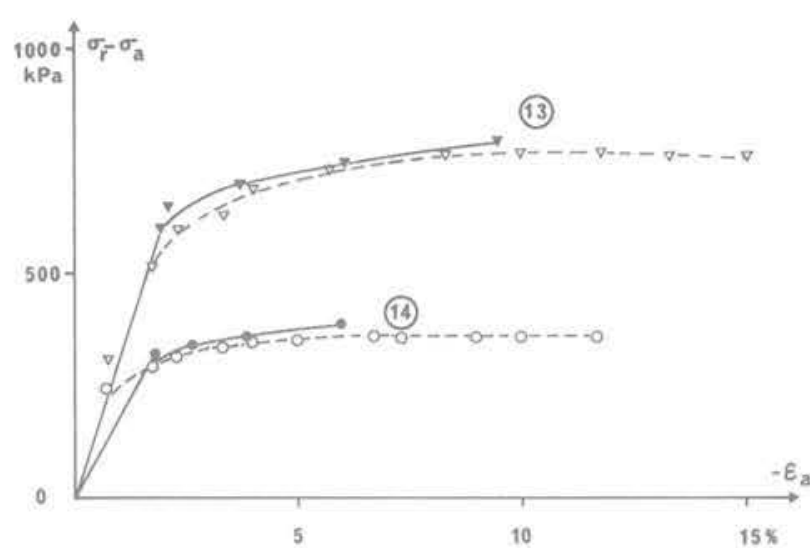

Fig. 18 Courbes déviateur des contraintes-tassement, pour les essais 13 et 14 sur des billes de plomb. Comparaison des résultats calculés (en noir) et des résultats expérimentaux (en clair)

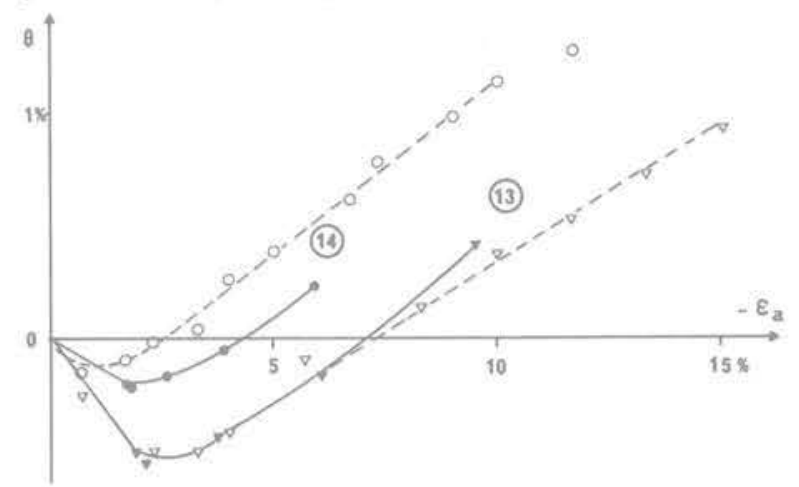

Fig. 19 Courbes variation de volume-tassement, pour les essais 13 et 14 sur des billes de plomb. Comparaison des résultats calculés (en noir) et des résultats expérimentaux (en clair)

Fig. 21 Courbes variation de volume-tassement, pour les essais 46, 52, 53 et 44 sur des billes de plomb. Comparaison des résultats calculés (en noir) et des résultats expérimentaux (en clair)

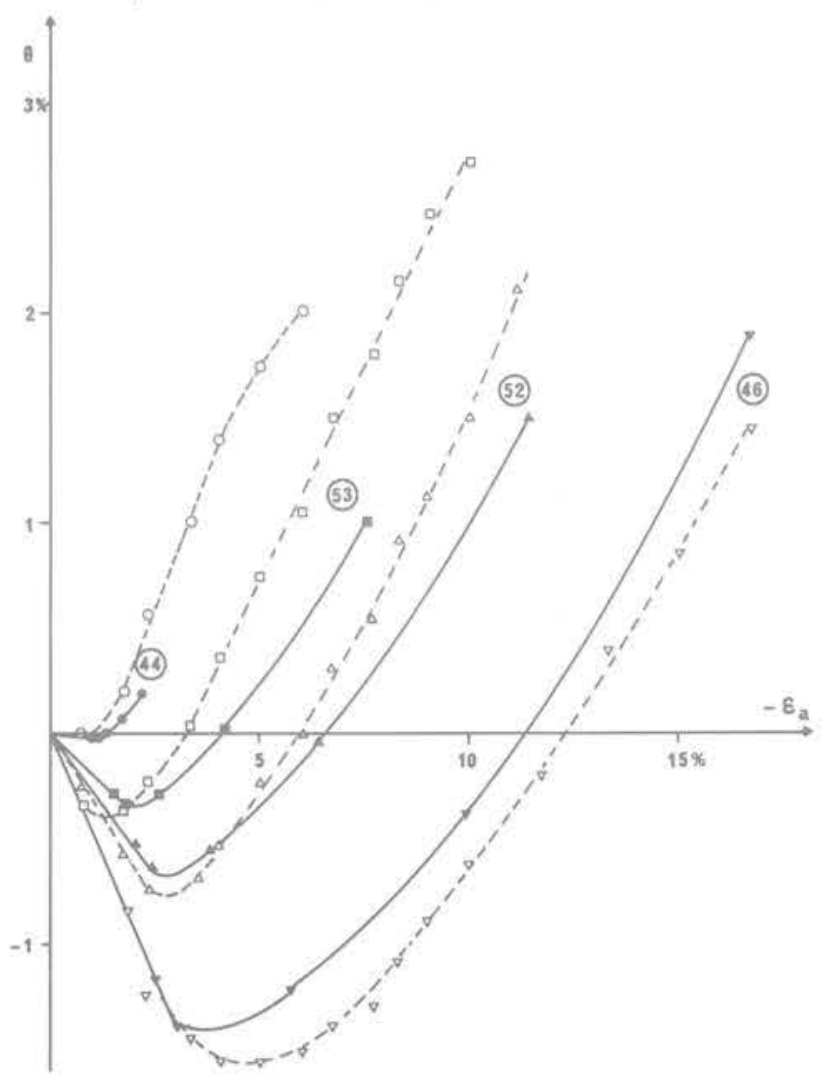




\begin{tabular}{|c|c|c|c|c|c|c|c|c|c|}
\hline $\begin{array}{ll}\text { Matériau } & \\
& 1 \\
& \end{array}$ & $\begin{array}{ll}\text { Essai } & \\
& 2 \\
& \end{array}$ & 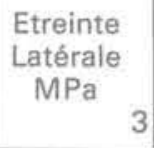 & \begin{tabular}{|c|}
\multicolumn{2}{c}{ Poids } \\
Volumique \\
$\mathrm{kN} / \mathrm{m}^{3}$ \\
\\
\end{tabular} & 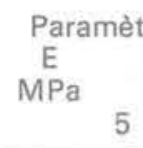 & $\begin{array}{l}\text { es de la lo } \\
6 \\
6\end{array}$ & \begin{tabular}{cc} 
de compo \\
\multicolumn{1}{c}{} \\
degré \\
\\
& 7
\end{tabular} & $\begin{array}{l}\text { ement } \\
\varphi_{\mu} \\
\text { degré } \\
\end{array}$ & $\begin{array}{c}\varphi^{\prime \prime} \\
\text { degré } \\
\end{array}$ & ${ }^{\varphi-\varphi^{\prime \prime}}{ }_{10}^{\text {degré }}$ \\
\hline $\begin{array}{l}\text { Billes de } \\
\text { plomb } \\
n=39 \%\end{array}$ & $\begin{array}{r}19 \\
25 \\
4 \\
40\end{array}$ & $\begin{array}{l}0,552 \\
0,276 \\
0,138 \\
0,052\end{array}$ & $\begin{array}{l}62,2 \\
62,2 \\
62,2 \\
62,2\end{array}$ & $\begin{array}{l}60 \\
34 \\
34 \\
20\end{array}$ & $\begin{array}{l}0,31 \\
0,41 \\
0,48 \\
0,49\end{array}$ & $\begin{array}{l}40,7 \\
40,7 \\
40,7 \\
40,7\end{array}$ & $\begin{array}{l}29,5 \\
29,5 \\
29,5 \\
29,5\end{array}$ & $\begin{array}{l}12,0 \\
12,0 \\
12,0 \\
12,0\end{array}$ & $\begin{array}{l}28,7 \\
28,7 \\
28,7 \\
28,7\end{array}$ \\
\hline $\begin{array}{l}\text { Billes de } \\
\text { plomb } \\
\text { lubrifiées } \\
n=39 \%\end{array}$ & $\begin{array}{l}13 \\
14\end{array}$ & $\begin{array}{l}0,552 \\
0,276\end{array}$ & $\begin{array}{l}62,2 \\
62,2\end{array}$ & $\begin{array}{l}31 \\
18\end{array}$ & $\begin{array}{l}0,37 \\
0,44\end{array}$ & $\begin{array}{l}24,1 \\
24,1\end{array}$ & $\begin{array}{l}20,5 \\
20,5\end{array}$ & $\begin{array}{l}2,6 \\
2,6\end{array}$ & $\begin{array}{l}21,5 \\
21,5\end{array}$ \\
\hline $\begin{array}{l}\text { Billes de } \\
\text { plomb } \\
n=43 \%\end{array}$ & $\begin{array}{l}46 \\
52 \\
53 \\
44\end{array}$ & $\begin{array}{l}0,552 \\
0,276 \\
0,138 \\
0,052\end{array}$ & $\begin{array}{l}58,2 \\
58,2 \\
58,2 \\
58,2\end{array}$ & $\begin{array}{l}40 \\
25 \\
17 \\
12\end{array}$ & $\begin{array}{l}0,27 \\
0,37 \\
0,41 \\
0,49\end{array}$ & $\begin{array}{l}37,1 \\
37,1 \\
37,1 \\
37,1\end{array}$ & $\begin{array}{l}29,5 \\
29,5 \\
29,5 \\
29,5\end{array}$ & $\begin{array}{l}7,2 \\
7,2 \\
7,2 \\
7,2\end{array}$ & $\begin{array}{l}29,9 \\
29,9 \\
29,9 \\
29,9\end{array}$ \\
\hline Sable & $\begin{array}{l}1 \\
2 \\
3\end{array}$ & $\begin{array}{l}0,294 \\
0,196 \\
0,098\end{array}$ & $\begin{array}{l}14,7 \\
14,7 \\
14,7\end{array}$ & $\begin{array}{l}36 \\
32 \\
14\end{array}$ & $\begin{array}{l}0,37 \\
0,37 \\
0,41\end{array}$ & $\begin{array}{l}39,0 \\
39,0 \\
39,0\end{array}$ & $\begin{array}{l}28,0 \\
28,0 \\
28,0\end{array}$ & $\begin{array}{l}8,3 \\
8,3 \\
8,3\end{array}$ & $\begin{array}{l}30,7 \\
30,7 \\
30,7\end{array}$ \\
\hline
\end{tabular}

Fig. 22 Tableau des paramètres de la loi de comportement

\subsection{Valeur des paramètres utilisés}

Le tableau 22 regroupe, dans les colonnes $5,6,7$ et 8 . l'ensemble des paramètres utilisés pour les calculs par ordinateur. Nous remarquons en particulier la coïncidence qui existe entre les valeurs $\varphi_{\mu}$ introduites dans le programme et la différence $\varphi-\varphi$ " de la colonne $10(\varphi$ frottement ultime du matériau, et $\varphi^{\prime \prime}$ angle du critère d'écoulement non associé). Nous avons étudié sur la figure 23 la relation qui existe entre ces angles. Nous voyons que la droite tiretée, qui suppose l'égalité des valeurs, passe au milieu des points. Nous considérons donc que l'égalité (11) est vérifiée. Ceci permet d'éliminer le paramètre $\varphi^{\prime \prime}$ de la loi de comportement. Les seuls paramètres restant sont donc $E$ module de Young, $v$ coefficient de Poisson, $\varphi$ frottement ultime, $\varphi_{\mu}$ frottement grain sur grain.

E exprime la « raideur » du sol

$v$ détermine la contraction volumique du sol quand on le charge

$\varphi$. est l'angle de frottement de la rupture

$\varphi_{\mu}$ définit le seuil d'écrouissage

Newland et al. ont mesuré le frottement intergranulaire de leur échantillon de billes de plomb. Ils ont aussi mesuré l'angle de frottement correspondant au palier de contrainte obtenue une fois que l'on a dépassé le pic du déviateur caractérisant la rupture (ils l'ont appelé $\varphi_{\mathrm{f}}$ ).Dans le cas de l'échantillon normal $\varphi_{f}$ et $\varphi_{\mu}$ mesuré sont égaux à $30^{\circ}$ et pratiquement identique au $\varphi_{\mu}$ du calcul (29०5). Dans le cas de l'échantillon lubrifié $\varphi_{f}\left(22^{\circ}\right)$ est proche de $\varphi_{\mu}$ du calcul $\left(20^{\circ} 5\right)$ mais par contre $\varphi_{\mu}$ mesuré est très faible $\left(8^{\circ} 5\right)$.

D'une façon générale, on peut dire que les calculs permettent de retrouver les expériences triaxiales.
Fig. 23 Comparaison entre la valeur du frottement grain sur grain et la valeur du décalage angulaire du vecteur déformation plastique à la rupture

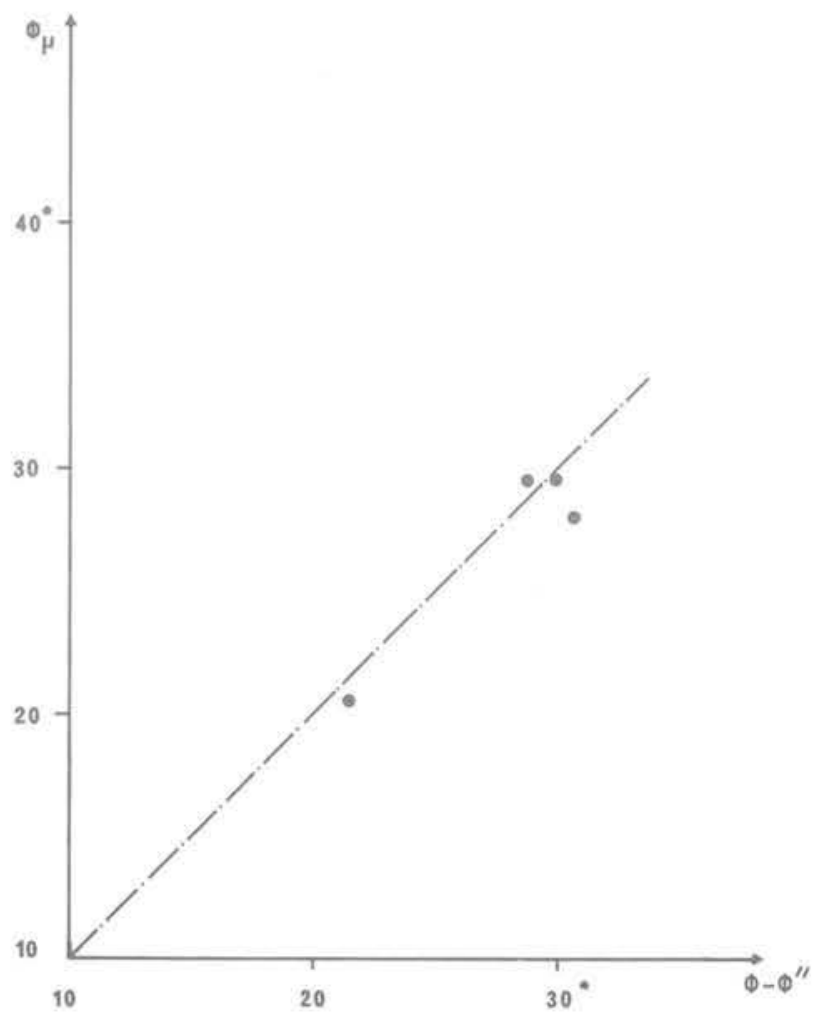




\section{Conclusion}

Les applications de la méthode des éléments finis aux calculs de mécanique des sols nécessitent la connaissance d'une loi de comportement du milieu étudié.

Nous avons donc mis au point une méthode de calcul prenant en compte l'écrouissage et la plasticité non standard du sol. Cette loi de comportement, issue des travaux de Frydman et al (1973) présente par rapport aux lois actuellement utilisées les avantages suivants :

- elle a une formation théorique exacte

- elle nécessite de définir le milieu pulvérulent par quatre paramètres seulement $\left(E, v, \varphi\right.$ et $\left.\varphi_{\mu}\right)$, dont la définition est simple

- elle prend en compte la dilatance du sol

- elle est globale et nécessite par conséquent moins de temps de calcul sur ordinateur qu'une méthode incrémentale.

L'application de cette loi par la méthode des éléments finis au cas de l'essai triaxial fait apparaitre une très bonne concordance entre les résultats calculés et les résultats expérimentaux. Cette constatation permet de conclure à la validité de la loi proposée.

La méthode de calcul du comportement du sol apparaît comme un moyen de calcul simple et sûr, mais elle limitée pour l'instant aux sols pulvérulents denses. Elle devrait permettre de proposer prochainement des solutions théoriques exactes à des problèmes de Génie Civil (soutènements, fondations, pentes, essai pressiométrique).

\section{Remerciement :}

Nous tenons à remercier M. Guellec pour les remarques très intéressantes qu'il nous a fournies pour la rédaction de cet article et pour l'utilisation du programme ROSALIE (Guellec - 1978).

\section{Références Bibllographiques}

[1] BARDEN et KHAYATT - Incremental strain rate ratios an strengh of sand in the triaxial test. Geotechnique Décembre 1966 - pp. 338-357

[2] BENT HANSEN - Line ruptures regarded as narrow rupture zone C.R. Conf. sur les problèmes de poussée des terres. Bruxelles 1958 - Vol. 1 - pp. 39-49.

[3] BISHOP - The strengh of soils as engineering material. Geotechnique - June 72 - pp. 91-128

[4] CHAMBON - Applications de la M.E.F. et d'une loi rhéologique incrémentale aux calculs de mécanique des sols. Thèse Doct. Ingénieur - Grenoble 1975

[5] DARMAR et PLESSIET - Etude de l'essai triaxial. Rapport interne à I'I.N.S.A. de LYON - 1964.

[6] DARVE - Contribution à la détermination de la loi rhéologique incrémentale des sols. Thèse Doct. Ingénieur Grenoble 1974
[7] DRUCKER, GIBSON, HENKEL - Soil mechanics and work hardening theories of plasticity. Proc. Am. Soc. civ. eng. $1955-81$ separate pp. $798.1-798.14$.

[8] DRUCKER, PRAGER - Soil mechanics and plastic analysis or limit design. Q. Appl. Math. - Vol. 2 (52), pp. 157-165

[9] DRUCKER, PRAGER, FREENBERG - Extended limit theorems for continuous media. Q. Appl. Math, - Vol. 9, pp. 381-389.

[10] DUNCAN, CHANG - Non linear analysis of stress and strain in soils. ASCE Soil mech. - Sept. 70, pp. 1629-1653.

[11] FRYDMAN - The strain hardening behaviour of particulate media. Can. Geot. J. - 1976, num. 13, pp. 311-323.

[12] FRYDMAN - Yielding of sans in plane strain. ASCE Soil mech. - May 74, pp. 491-501.

[13] FRYDMAN, ZEITLEN, ALPAN - The yielding behaviour of particulate media. Can. Geot. J. 10 - 1973, pp. 341362.

[14] GUELLEC, Correspondance privée - 1978

[15] GUELLEC - ROSALIE : Système de calcul des massifs et des structures - Section des modèles numériques no 1.09.14.6. Laboratoire Central des Ponts et Chaussées 1976.

[16] ISMAEL, KIRKPATRICK - Generalised plastic potentiel function for sands. First Baltic Conf. - GDANSK 1975 Vol. 2, pp. 249-257.

[17] KIRKPATRICK - The condition of failure for sands. 4 int. conf. soil mech. - 1957, pp. 172-178.

[18] LADE, DUNCAN - Elastoplastic stress - strain theory for cohesionless soil. ASCE Soil mech. - Oct. 75, pp. 1037 1053.

[19] MONNET - Détermination d'une loi d'écrouissage des sols et utilisation par la méthode des éléments finis. Thèse Doct. Ingénieur - INSA de LYON - 1977.

[20] NADAI - Theory of flow and fracture of solid. Vol. 2 Mac Graw Hill - 1963, pp. 46-47

[21] NAGARAJ, SOMASHEKAR - The failure criteria for soils. Can Geot. J. - Vol. 2 - 74, pp. 628-632.

[22] NEWLAND et ALLELY - Volumes changes in drained triaxial tests on granular materials. Géotechnique - 1957 pp. $17-34$

[23] POOROOSHASB, HOLUBEC, SHERBOURNE Yielding and flow of sand in triaxial compression part 1. Can. Geot. J. 1966 - Vol. 3, N, 4, pp. 179-190

[24] POOROOSHASB, HOLUBEC, SHERBOURNE - Yie/ding and flow of sand in triaxial compression part 2 and 3. Can. Geot. J. Nov. 67 - pp. 376-397.

[25] RADENKOVIC - Théorèmes limites pour un matériau de Coulomb à dilatation non standardisée. C.R. Ac Sc. Paris 1961, 252, pp, 4103-4104.

[26] ROWE - The stress dilatancy relation for static equilibrium. Proc. Roy. Soc. - 1962 - 269 pp. 500-527.

[27] SALENCON - Théorie de la plasticité pour les applications à la mécanique des sols. Editions Eyrolles - 1974.

[28] SCHOFIELD, WROTH - Critical state soil mechanics. Mac Graw Hill - 1958.

[29] ZIENKIEWICZ - La méthode des éléments finis. Editions Edisciences - 1973. 